\title{
Fluid Cells Fabrication in Sol-Gel and Porous Glass
}

\author{
Yaroslava M. Andreeva ${ }^{1}$, Maksim M. Sergeev ${ }^{1}$, Roman A. Zakoldaev ${ }^{1, *}$, Uliana E. Gabysheva ${ }^{1}$, Vadim P. Veiko ${ }^{1}$, Evgeniy B. \\ Yakovlev $^{1}$, Sergey I. Kudryashov ${ }^{1,2}$, Pavel A. Danilov², Andrey A. Ionin², Francis Vocanson ${ }^{3}$, Tatiana E. Itina ${ }^{1,3}$, Tatiana V. \\ Antropova $^{4}$, Oleg S. Medvedev ${ }^{5}$ \\ ${ }^{1}$ ITMO University, 49, Kronverkskiy av., 197101, St. Petersburg, Russia \\ ${ }^{2}$ Lebedev Physical Institute, 53, Leninsky prospect, 119991, Moscow, Russia \\ ${ }^{3}$ Hubert Curien Laboratory, UMR CNRS 5516/UJM/University of Lyon, Bat. F, 18 rue du Pr. Benoit Lauras, 42000 Saint- \\ Etienne, France \\ ${ }^{4}$ Grebenshchikov Institute of Silicate Chemistry, Russian Academy of Sciences, 2 Makarova nab., 199034, St. Petersburg, \\ Russia \\ ${ }^{5}$ St.-Petersburg State University, Research Park, IRC for Nanotechnology, Ulianovskaya str. 1, 198504, St. Petersburg, \\ Russia
}

\section{E-mail: zakoldaev@gmail.com}

We investigate laser treatment regimes suitable for the fabrication of the isolated cells in a porous silica glass and a sol-gel film. Three-dimensional structures are produced as a result of the porous glass densification induced by femtosecond laser pulses. We also demonstrate that $\mathrm{CO}_{2}$ laser can be used to form such structures in the sol-gel film deposited on the glass. In the porous glass, barriers with various permeability were created, which were used as walls in fluid cells. Laserinduced microrelief formation on the surface of the sol-gel film provides isolation for the separated porous cells. The impermeability of porous cells is tested by impregnation them with different dyes (rhodamine 6G, fuchsine, etc.).

DOI: 10.2961/ilmn.2018.03.0008

Keywords: $\mathrm{CO}_{2}$-laser irradiation, femtosecond laser pulses, molecular barrier, fluid cell, porous glass, sol-gel film.

\section{Introduction}

Nowadays, porous silica glass is of a great interest as a solid-state medium for various agents in microfluidics and analytics devices [1]. Functional elements for photonics are fabricated by using a porous glass (PG) or a sol-gel (SG) impregnated with various organic agents [2], salts [3] and metal nanoparticles [4]. Operating principle of such elements is based on the effect of plasmon resonance [5], fluorescence [6], photoelectric and magnetic nanoparticle properties, as well as photochemical reactions [7]. Laser processing of PG and SG allows the formation of various submicron and nanoscale structures by localized control the density of the porous medium. There are several advantages of the PG, among which their transparency in visible and near IR ranges, chemical and biological durability and the simplicity of impregnating them with various liquid agents. Despite their attractive properties, these materials are still not commonly used in many applications because of the difficulty in their processing. Previous studies have already revealed major mechanisms of femtosecond laser irradiation of $\mathrm{PG}$ and fabrication of complex three-dimensional fluidic systems composed of hollow micro- and nanostructures $[8,9]$. However, there is no any attempt to fabricate of monolitic structures (in view of densified trcks) for the entire thickness of the sample. Thus, the need for local control over medium porosity, meaning the separation into independent porous cells via fabrication of through barrier, is a topical issue. The solution to this problem will allow the application of PG and SG for integral systems and multifunctional (multileveled) sensors fabrication [7].

In this work, different methods of laser-induced formation of insulating barriers in porous materials are presented. A combination of two techniques has allowed us to fabricate a monolithic densified structure, which divides porous glass in independent cells. The application of $\mathrm{CO}_{2}$ laser is not enough because the absorption mechanism allows us to change density only on the surface or at a pre-surface layer. That is why femtosecond laser pulses have been used in the experiments to achieve an inner densification of the porous glass. The advantage of the $\mathrm{CO}_{2}$ laser is that it can easily induce surface densification, whereas femtosecond laser pulses often damage surface layer. The limitation of the $\mathrm{CO}_{2}$ laser is that it cannot be used for inner densification of the sample.

In case of sol-gel only $\mathrm{CO}_{2}$-laser irradiation is applied to fabricate hydrophobic relief. As for PG, the fabrication of through barrier is performed by combination of femtosecond laser irradiation for inner densification and $\mathrm{CO}_{2}-$ laser irradiation for surface densification of the barrier.

The tests of fabricated structures show that each cell surrounded by the barriers can operate independently.

\section{Experiments and Methods}

In this work two types of porous matrices: PG with a thickness of $1.5 \mathrm{~mm}$, average pore size $\sim 4 \mathrm{~nm}$, porosity $28 \%$ and SG with a thickness of $180 \mathrm{~nm}$, average pore size $\sim 7.5 \mathrm{~nm}$, porosity $10 \%$ are used. PG is fabricated by 
through etching of sodium borosilicate glass [10]. SG is produced by sol-gel synthesis and dip coated on soda-limesilica glass substrate (see [11]). These porous medium possess high transparency $(\sim 90 \%)$ in the visible and near IR ranges $(0.4-1.1 \mu \mathrm{m})$, and the average refraction index $n$ is about 1.34 in the visible spectral range.

The fabrication of hydrophobic relief containing liquid on the surface of SG and surface densification of PG were carried out using $\mathrm{CO}_{2}$-laser irradiation, which is strongly absorbed by $\mathrm{SiO}_{2}$ bonds. In the experimental setup, $\mathrm{CO}_{2}$ laser beam (TEM ${ }_{00}, D=3.5 \mathrm{~mm}, \theta=4.0 \mathrm{mrad}$ ) was focused on the surface of the target by a Ge-objective with a focal length $\mathrm{F}=25 \mathrm{~mm}$. Irradiation was conducted in repetitively-pulsed laser scanning regime ( $\tau=200 \mu \mathrm{s}, f=1 / \tau)$.

Localized modification of PG structure was carried out by ultrashort laser pulses. The method description and experimental details are given in Ref. [12]. An objective $(10 \mathrm{X}, 0.25)$ focused the laser beam into the focal spot of $2 \omega_{0} \sim 3.5 \mu \mathrm{m}$ in $1 / \mathrm{e}$-diameter. Densification means pore collapse in the focusing region of the laser beam. Stairstep order of densified lines in the whole width of the PG plate allowed the organization of a wall, or a so-called "barrier". The permeability of the barrier was controlled by the step between densified lines. The organization of porous cells in used porous matrices was provided by orthogonal positioning of the barriers. Then, the produced cells were impregnated by rhodamine $6 \mathrm{G}$ and different salts solutions in order to test their impermeability.

The fabricated microstructures were examined by optical microscopy (Carl Zeiss) in the transmitted and reflected light. The microrelief depth was measured via profilometry (Hommel Tester T8000). Additionally, nanostructures formed in the porous glass were also investigated by scanning electronic microscopy (SEM Carl Zeiss Merlin).

\section{Results and discussion}

\subsection{Insulating barriers in SG under $\mathrm{CO}_{2}$-laser action}

The fabrication of insulated barriers in SG were performed by $\mathrm{CO}_{2}$ laser irradiation with a laser beam radius of $\omega_{0}=26.5 \mu \mathrm{m}$ (fig. $1 \mathrm{a}, \mathrm{b}$ ). The obtained results demonstrate a microrelief with complex shape (fig. $1 \mathrm{c}$ ) characterized by different depth and width was formed as a result of direct laser writing at the different scanning speed $v=100-700$ $\mu \mathrm{m} / \mathrm{s}$ and laser power in the range of $\mathrm{P}=100-400 \mathrm{~mW}$ (fig. $1 \mathrm{a}, \mathrm{c}$ ).

$\mathrm{CO}_{2}$ laser irradiation is absorbed mainly by the glass substrate due to the small thickness of SG $(180 \mathrm{~nm})$. Thus, the appearance of the relief in the SG surface is related to the laser-induced heating and modification of the substrate. The absorption of SG and substrate can be estimated via Beer-Lambert law taking into account the Fresnel reflection for the normal incidence of laser beam [13] as follows:

$$
A(z)=\left[1-\left(\frac{n_{2}-n_{1}}{n_{2}+n_{1}}\right)^{2}\right]\left[1-\exp \left(-\frac{4 \pi n_{2} \kappa_{2}}{\lambda} z\right)\right],
$$

where $n_{1 / 2}$ and $\kappa_{1 / 2}$ are refractive and absorption indices for the first (SG) $\left(\mathrm{n}_{1}=1.96, \mathrm{k}_{1}=0.08\right)$ and the second (substrate) $\left(\mathrm{n}_{2}=1.97, \mathrm{k}_{2}=0.31\right)$ media, $\lambda=10.6 \mu \mathrm{m}$ is laser wavelength, $\mathrm{z}-$ the depth.

Therefore, the contribution of SG absorption is only $A_{f}$ $\sim 3 \%$ and the proportion of reflected irradiation $R_{f} \sim 12 \%$ for the films with thickness $<<\lambda$. The reflection on the film-substrate interface is negligibly small due to insignificant difference of refractive indices between two media $(\Delta n=0.01)$. Thus, the contribution of the substrate absorption is $A_{s} \sim 85 \%$. Considering the optical losses in $\mathrm{SG}$ the penetration depth of $\mathrm{CO}_{2}$ laser radiation in the substrate (for intensity level $1 / \mathrm{e}$ ) is estimated according to the equation [14]:

$$
h_{s} \approx \frac{\lambda}{4 \pi n_{s} \kappa_{s}} \ln \left[\frac{1}{A_{f}+1 / e}\right]
$$

The average light penetration depth in the substrate is $\delta=1.45 \mu \mathrm{m}$ including light passing through two media (fig. 1d), which is less than the diameter of the laser beam $\left(2 \omega_{0}>>\delta\right)$

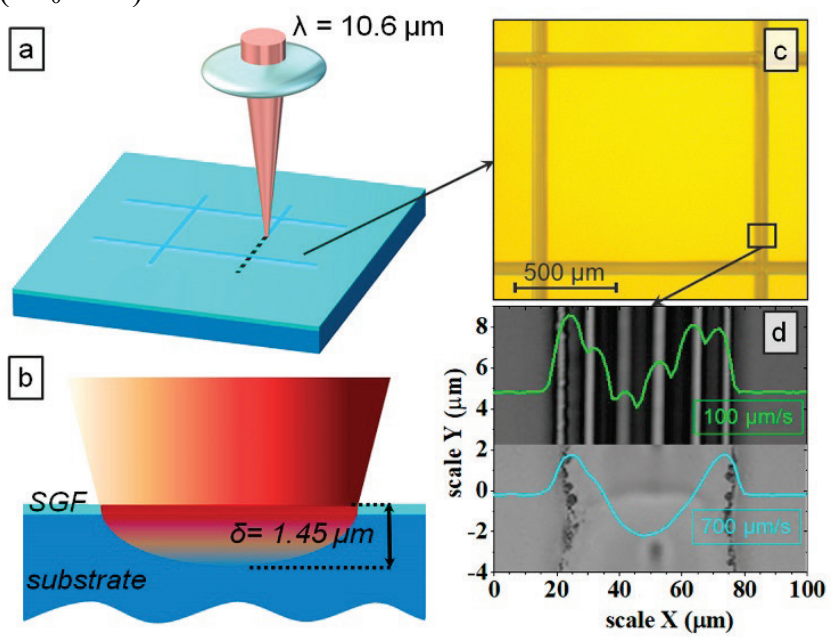

Fig. 1 The schematic view of the barrier procedure fabrication in SG: direct $\mathrm{CO}_{2}$-laser writing (a); Illustration of laser radiation absorption by two mediums (b). Optical microimage of isolated porous cell (c). Profilometry of formed barriers $\mathrm{P}=190 \mathrm{~mW}$ and different scanning speeds $(v=100$ and $700 \mu \mathrm{m} / \mathrm{s})(\mathrm{d})$

In case of laser-induced modification of SG the difference between the temperature of the $\mathrm{SiO}_{2}$ film densification $T_{d} \sim 750{ }^{\circ} \mathrm{C}[15]$ and the softening temperature of the substrate $\left(T_{s} \sim 550^{\circ} \mathrm{C}\right)[16]$ is essential. Moreover, thermal expansion coefficients for the materials are different and equal to $\alpha_{f}=0.55 \cdot 10^{-6} \mathrm{~K}^{-1}$ for SG and $\alpha_{s}=9.10 \cdot 10^{-6} \mathrm{~K}^{-1}$ for substrate.

$\mathrm{CO}_{2}$ laser irradiation leads to formation of the softened area in glass with depth of $d_{t h}=\delta+2(a \tau)^{0.5}=11.4 \mu \mathrm{m}$ and width of $2 \omega_{0}+2(a \tau)^{0.5}=73 \mu \mathrm{m}$, where $a$ is thermal diffusivity, $\tau$ is pulse duration. Thermal expansion causes the material surface to rise. In addition, high temperature gradients force the material redeployment from the center of laser irradiated area to its periphery, where the temperature is significantly lower.

To estimate the temperature distribution in the laser affected zone the heat equation for linearly moving heat source is applied [17].

To solve the equation the following assumptions are taken into account: 
- thermal conductivity $k_{s}=1.1 \mathrm{~W} / \mathrm{mK}$, thermal diffusivity $a_{s}=0.5 \cdot 10^{-6} \mathrm{~m}^{2} / \mathrm{s}$ and absorptance $A=[1-$ $\left.\left(\left(n_{f}-1\right) /\left(n_{f}+1\right)\right)^{2}\right]$ of the substrate are not depend on the temperature;

- the contribution of convective heat transfer does not exceed the contribution of conductive heat transfer and keeps lower than $A_{\text {loss }}=0.23$;

- surface heat source i.e. $\delta<\lambda<<H$, where $H$ is the substrate thickness.

Thus, the heat equation for stationary heating mode can be calculated (3). The heating source movement is along $\mathrm{OX}$, the heat distribution is along $\mathrm{OZ}$.

$$
\begin{aligned}
& T(x, y, z)=\left(1-A_{\text {loss }}\right)\left[1-\left(\frac{n_{f}-1}{n_{f}+1}\right)^{2}\right] \cdot \int_{0}^{2 \pi} \int_{0}^{\infty} \frac{P}{\left(\pi \omega_{0}\right)^{2} k_{s}} \\
& \times \exp \left[-\frac{v r}{2 a_{s}} \cdot \cos \varphi-2 \frac{(x-r \cdot \cos \varphi)^{2}+(y-r \cdot \sin \varphi)^{2}}{\omega_{0}^{2}}\right] . \\
& \times \frac{r}{\sqrt{r^{2}+(z+\delta)^{2}}} \cdot \exp \left[-\frac{v}{2 a_{s}} \sqrt{r^{2}+(z+\delta)^{2}}\right] d r d \varphi+T_{0}
\end{aligned}
$$

The microrelief was fabricated at laser power density range q from 7.7 to $10.3 \mathrm{~kW} / \mathrm{cm}^{2}$. The laser scanning speed $v$ was varied from 100 to $700 \mu \mathrm{m} / \mathrm{s}$. An increased power density $\left(>12.5 \mathrm{~kW} / \mathrm{cm}^{2}\right)$ led to the glass destruction. The temperature was estimated in 4 different points: in the center of the laser spot on the surface; in the maximum of the relief at $\omega_{01}$ from the center on the surface; at the periphery of the laser track $d_{t h}$ on the surface; and in the center of laser spot at a depth of $\omega_{01}$. Results of estimation are presented in table 1 . The temperature region corresponding to the softening of soda-lime glass is highlighted by purple and the blue regions highlighted show the temperature of viscous flow. Note that for red region the temperature increase leads to the destruction of the material.

Table 1. Estimated temperature for used parameters of laser treatment

\begin{tabular}{c|c|c|c|c|c}
\hline \multirow{2}{*}{$\begin{array}{c}q \\
\mathrm{~kW} / \mathrm{cm}^{2}\end{array}$} & \multirow{2}{*}{\begin{tabular}{c}
$\mu \mathrm{m} / \mathrm{s}$ \\
\cline { 3 - 6 }
\end{tabular}} & $T(0,0,0)$ & $T\left(0,0, h_{t h}\right)$ & $T\left(0, \omega_{01}, 0\right)$ & $T\left(0, d_{t h} / 2,0\right)$ \\
\cline { 2 - 6 } & 700 & 1665 & 968 & 620 & 580 \\
\cline { 2 - 6 } & 100 & 1676 & 980 & 630 & 590 \\
\hline \multirow{2}{*}{10.3} & 700 & 2200 & 1275 & 812 & 760 \\
\cline { 2 - 6 } & 100 & 2215 & 1290 & 827 & 775 \\
\hline \multirow{2}{*}{12.6} & 700 & 2700 & 1562 & 992 & 930 \\
\cline { 2 - 6 } & 100 & 2718 & 1581 & 1011 & 947 \\
\hline
\end{tabular}

The temperature gradient along OY which is $d T / d y=\left[T(0,0,0)-T\left(0, \omega_{01}, 0\right)\right] / \omega_{01}$, is estimated using table 1 . The barriers fabrication is accompanied by the gradient values of $40-50{ }^{\circ} \mathrm{C} / \mu \mathrm{m}$ (fig. 2). Further increasing of heating rate up to $60{ }^{\circ} \mathrm{C} / \mu \mathrm{m}$ was related to the material destruction. The temperature gradient along $\mathrm{OZ}$ $d T / d z=\left[T(0,0,0)-T\left(0,0, h_{t h}\right)\right] / h_{t h}$ in case of barriers formation was $60-80{ }^{\circ} \mathrm{C} / \mu \mathrm{m}$ and destruction occurs at $d T / d z=100^{\circ} \mathrm{C} / \mu \mathrm{m}$.

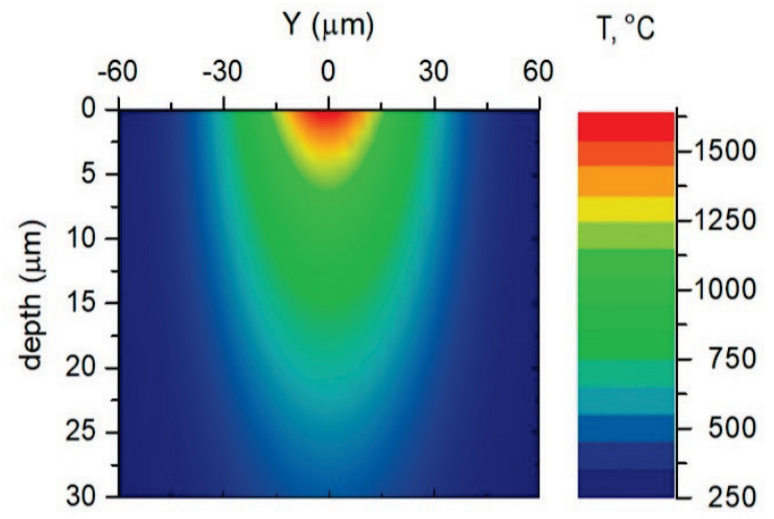

Fig. 2 Modeling of temperature distribution in soda-lime glass substrate irradiated by $\mathrm{CO}_{2}$ laser $\left(\mathrm{q}=7.7 \mathrm{~kW} / \mathrm{cm}^{2}, v=100 \mu \mathrm{m} / \mathrm{s}\right)$. Note that the presence of SG is neglected due to its small thickness.

Modification of SG on the soda-lime glass substrate is related not to only temperature distribution but to time of heat action $t=2 \omega_{0} / v$. This value is maximal for $v=100 \mu \mathrm{m} / \mathrm{s}$ and reaches $1.37 \mathrm{~s}$. The temperature increase rate in the center of softened area $d T / d t=T\left(0,0, h_{t h}\right) / t_{\max }$ is estimated of $700-940{ }^{\circ} \mathrm{C} / \mathrm{s}$ during fabrication process. In case of material destruction this value is $1140{ }^{\circ} \mathrm{C} / \mathrm{s}$.

Therefore, the intensive local laser heating initiates the appearance of rather high structures (up to 3-4 $\mu \mathrm{m}$ ). Such a relief make it possible to isolate the porous cell which can be used for integral soaking (fig. $1 \mathrm{~b}, \mathrm{c}$ ).

To test the resulting barriers, a drop of $\mathrm{Cu}\left(\mathrm{NO}_{3}\right)_{2}$ of about $2 \mu 1$ was placed into the cell (fig. 3). Such fluid volume is enough to soak porous media within one cell. The barriers can hold the drop for relatively long time (until the drop dries up) thus the integral impregnation of the material can be implemented.

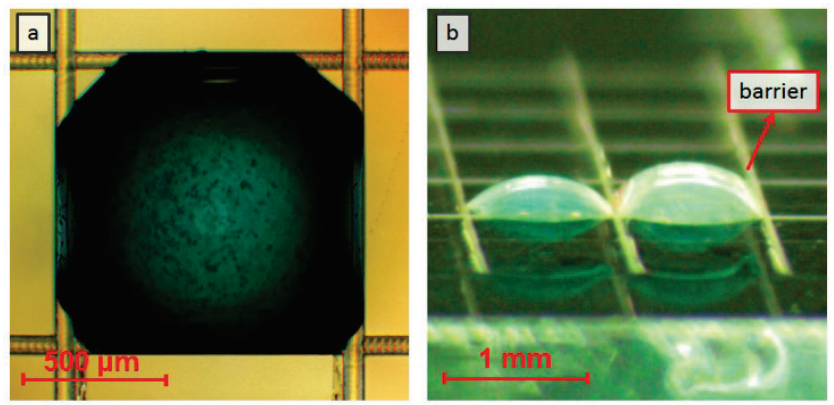

Fig. 3 Testing of barrier permittivity in SG: soaking with $\mathrm{Cu}\left(\mathrm{NO}_{3}\right)_{2}$ aq. $3 \mathrm{M}$ solution (a).

\subsection{Barriers with controlled permeability fabrication in PG}

Barriers were fabricated in PG in two stages (fig. 4). The inner modification means that a local pore collapse occurred after the multiphoton absorption of ultrashort laser pulses. High repetition rate (up to $0.5 \mathrm{MHz}$ ) provides the effect of heat accumulation in the beam waist region, providing a monolithic densification of the PG in the form of a line (fig. 4 a). However, the height of such structure is equal to $h=100-150 \mu \mathrm{m}$ and restricted by the focusing system.

Due to difficulties of ultrashort laser pulses machining in the border glass-air (scratches and cracks are occur), the following surface densification in the region of the formed 
inner barrier was accomplished by $\mathrm{CO}_{2}$ laser irradiation (fig. $4 \mathrm{a})$, that led to glass heating, viscosity fluctuation and pore collapsing. $\mathrm{CO}_{2}$-laser processing was carried out at a beam diameter $\sim 75 \mu \mathrm{m}$, scanning speed of $200 \mu \mathrm{m} / \mathrm{s}$ and a power of incident radiation $400 \mathrm{~mW}$. The densification was carried out at power density $\sim 2.1 \mathrm{~kW} / \mathrm{cm}^{2}$ (fig. $4 \mathrm{~b}$ ).

The penetration depth of $\mathrm{CO}_{2}$ laser radiation was estimated by using the expression (2) that gives $\delta=1.6 \mu \mathrm{m}$. However, taking into account the thermal diffusivity $a_{p g}=$ $0.5 \cdot 10^{-6} \mathrm{~m}^{2} / \mathrm{s}$, the height turned out to be equal to $166 \mu \mathrm{m}$. As a result of laser treatment, the density of material was locally changed from $\rho_{1}=1644 \mathrm{~kg} / \mathrm{m}^{3}$ up to the density of fused silica $\rho_{2}=2211 \mathrm{~kg} / \mathrm{m}^{3}$ [18]. Thus, a monolithic densified track (pore collapse can be seen in fig. 4 b) was formed on PG surface, its height was enough to be in a contact with inner densification (fig. $4 \mathrm{c}$ ), that ensuring the integrity of the barrier. In our study, the fabricated barrier can be used as a separator of molecules in a water solution of rhodamine $6 \mathrm{G}$, providing separation of rhodamine molecules (large in mass and size) from water molecules.

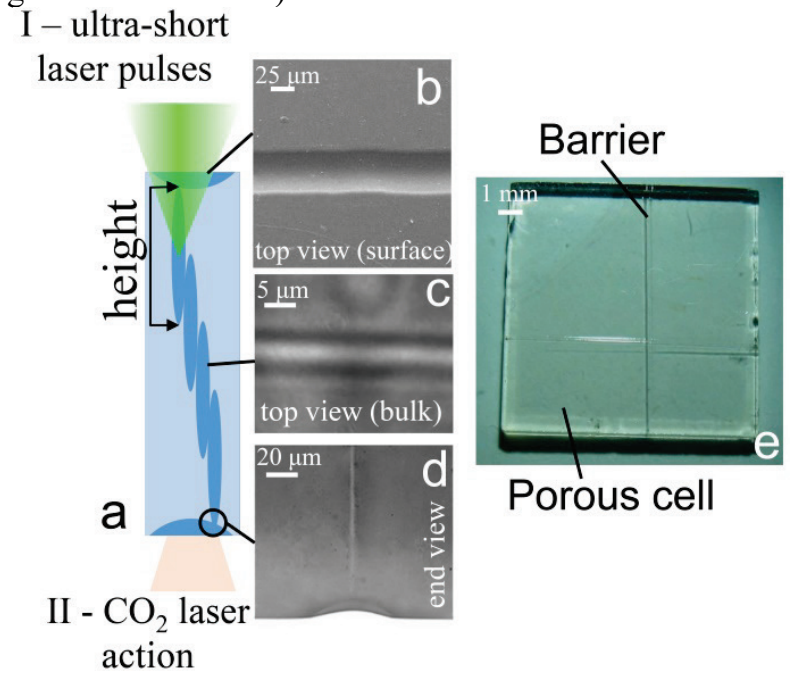

Fig. 4 Laser procedure for the barrier fabrication in PG plate: (i) inner densification PG by fs pulse action and (ii) surface densification by $\mathrm{CO}_{2}$ laser irradiation. The final structure: electron microscopy image of the surface (b), microscopy of the buried densified track (c) and the contact of inner and surface densification (d). Photo of isolated porous cells (e).

A few porous cells were fabricated in PG plate via orthogonal barriers displacement (fig. 4 e). The porous cell was soaked in water solution of rhodamine $6 \mathrm{G}$ for $10-15$ minutes (fig. 5 a) to fill the cell and test the permeability of the barrier. It can be clearly seen that rhodamine molecules were constrained by prescribed barriers, but at the same time, water molecules easily leaked through it (fig. $5 \mathrm{~b}$ ).

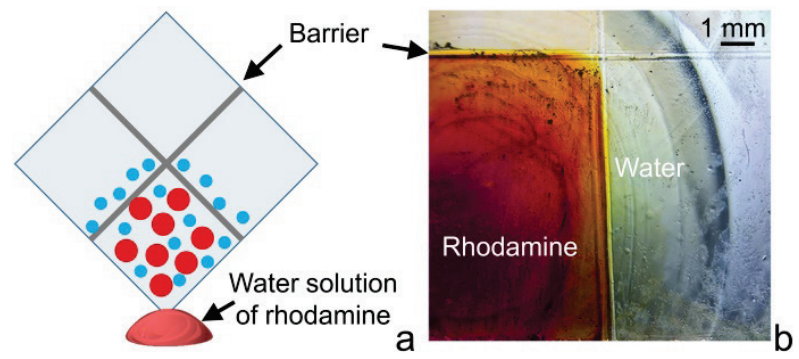

Fig. 5 The soaking of the porous cell with water solution of rhodamine 6G: schematic view (a) and photo (b)

\section{Conclusions}

In this work the features of laser modification mechanism of SG were considered. Conditions for laser processing of $\mathrm{SG}$ by $\mathrm{CO}_{2}$ laser radiation are determined. An estimation of the temperature fields providing a modification of the SG structure was carried out.

$\mathrm{CO}_{2}$ laser processing of SG was applied for fabrication of impenetrable barriers. The orthogonal displacement of such barriers allowed to organize isolated porous cells. Cells soaking confirmed their impenetrability.

Ultrashort laser pulses with following $\mathrm{CO}_{2}$ laser irradiation of PG plate were applied for barriers fabrication in PG. The permeability of the barrier was controlled by the step between inner densified lines. The barriers showed partial impermeability upon separation of the organic dye.

\section{Acknowledgments}

The reported study was financially supported by the Ministry of Education and Science of the Russian Federation, research agreement №14.587.21.0037 (RFMEFI58717X0037).

\section{References}

[1] F. He, Y. Liao, J. Lin, J. Song, L. Qiao, Y. Cheng and K. Sugioka: Sensors, 14, (2014) 19402.

[2] Y. Y. Maruo and J. Nakamura: Anal. Chim. Acta, 702, (2011) 247.

[3] T. Antropova, M. Girsova, I. Anfimova, I. Drozdova, I. Polyakova and N. Vedishcheva: J. Non-Cryst. Solids, 401, (2014) 139.

[4] A. Ciżman, K. Rogacki, E. Rysiakiewicz-Pasek, T. Antropova, O. Pshenko and R. Poprawski: J. Alloy. Compd., 649, (2015) 447.

[5] G.S. Andrei Stalmashonak, Amin Abdolvand: "UltraShort Pulsed Laser Engineered Metal-Glass Nanocomposites", (Springer International Publishing, 2013) pp. 70.

[6] C. Dey, M. Goswami and B. Karmakar: Mater. Res. Express, 2, (2015) 015014.

[7] A. Royon, Y. Petit, G. Papon, M. Richardson and L. Canioni: Opt. Mater. Express, 1, (2011) 866.

[8] Y. Liao and Y. Cheng: Micromachines, 5, (2014) 1106.

[9] Y. Liao, Y. Ju, L. Zhang, F. He, Q. Zhang, Y. Shen, D. Chen, Y. Cheng, Z. Xu and K. Sugioka: Opt. Lett., 35, (2010) 3225.

[10] V. Kreisberg and T. Antropova: Microporous Mesoporous Mater., 190, (2014) 128.

[11] Y. Battie, N. Destouches, F. Chassagneux, D. Jamon, L. Bois, N. Moncoffre and N. Toulhoat: Opt. Mater. Express, 1, (2011) 1019.

[12] V. P. Veiko, S. I. Kudryashov, M. M. Sergeev, R. A. Zakoldaev, P. A. Danilov, A. A. Ionin, T. V. Antropova and I. N. Anfimova: Laser Phys. Lett., 13, (2016) 055901.

[13] M. Born and E. Wolf: "Principles of optics: electromagnetic theory of propagation, interference and diffraction of light", (Cambridge University Press, Cambridge, 1999) p.865

[14] S. Gräf, G. Staupendahl, P. Gerling and F. A. Müller: J. Appl. Phys., 113, (2013) 013101.

[15] B. Fabes, D. Birnie and B. Zelinski: Thin Solid Films, 254, (1995) 175. 
[16] W. Martienssen and H. Warlimont: "Springer Handbook of Condensed Matter and Materials Data", (Springer, Berlin, 2005) pp. 1091.

[17] J. Cheng and A. Kar: J. Mat. Sci., 32, (1997) 6269.

[18] V.P. Veiko, E.B. Jakovlev and A.J. Nikiphorov: "Chemical processing of advanced materials" ed. by L.L. Hench, J.K. West, Chapter 82 (J. Wiley and Sons, New York, 1992) p. 919.

(Received: July 12, 2018, Accepted: October 7, 2018) 